\title{
BANDAS DE MACH EN RADIOLOGÍA
}

\author{
Drs. Pedro Melo C ${ }^{(1)}$, J. Antonio Escaffi J(2).
}

1. Becado Radiología, Facultad de Medicina Clínica Alemana, Universidad del Desarrollo. Chile

2. Radiólogo, Hospital Padre Hurtado y Clínica Las Condes. Chile

\section{MACH BANDS IN RADIOLOGY}

\begin{abstract}
Mach bands represent optical psychophysiological edge-enhancement phenomena perceived in different forms by different observers; they originate in adjacent areas of diverse density, giving rise to a thin black strip (negative Mach band) or a white strip (positive Mach band). They can be identified in simple radiographic examinations, including chest radiography, and also in CT topograms. Proper recognition of Mach bands helps to correctly interpret radiological examinations.
\end{abstract}

Keywords: Computed tomography, Mach bands, Radiography.

Resumen: Las bandas de Mach son fenómenos ópticos psicofisiológicos de realce de bordes que se perciben de forma muy variable entre los distintos observadores; se originan en áreas adyacentes de diferente densidad, dando origen a una fina banda negra o blanca, Mach negativo o positivo. Pueden identificarse en los exámenes radiológicos simples, como por ejemplo radiografía de tórax, y también en los topogramas de TC. El adecuado reconocimiento de las bandas de Mach ayuda a interpretar correctamente los exámenes radiológicos.

Palabras clave: Bandas de Mach, Radiografía, Tomografía computada.

\section{Introducción}

En ocasiones, en la percepción visual de la imagen radiológica se produce un fenómeno óptico denominado efecto Mach. Se percibe de forma muy variable entre los distintos observadores, en áreas adyacentes de diferente densidad donde se origina

Melo $\boldsymbol{P}$ y cols. Bandas de mach en radiología. Rev Chil de Radiol 2010; 16(2): 86-89.

Correspondencia: Dr. Pedro Melo Castillo

Dirección: Hospital Padre Hurtado. Santiago, Chile. pmelocastillo@gmail.com

Trabajo recibido el 05 de abril de 2010, aceptado para publicación el 18 de mayo de 2010. una fina banda negra (Mach negativo) o blanca (Mach positivo).

La primera descripción de este fenómeno corresponde a Ernst Mach, en 1865. Las bandas de Mach son fenómenos ópticos psicofisiológicos de realce de bordes ${ }^{(1)}$. Mach demostró que se observan bandas claras y oscuras en las proximidades de los contornos que separan las áreas claras y oscuras, aunque estas bandas no estén físicamente presentes (Figura 1).

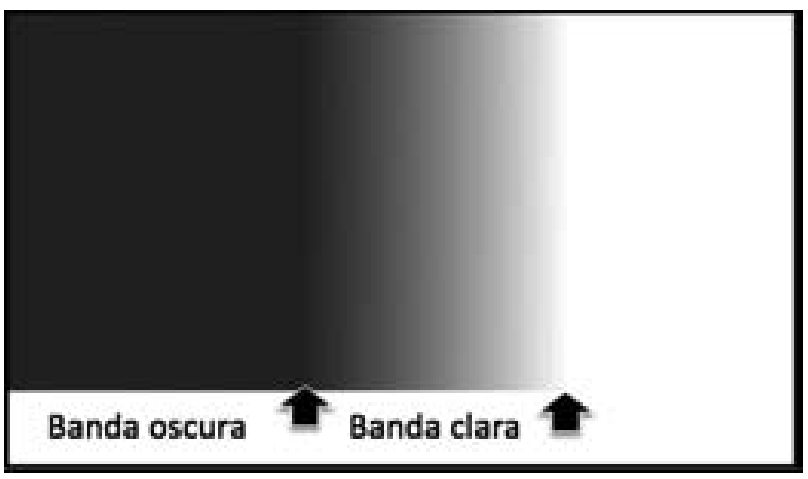

Figura 1. En una gráfica de escala de grises se observa una banda clara y otra oscura, que no corresponden a líneas reales.

La visualización de estas bandas dependerá del contorno de la estructura observada y la densidad óptica relativa de ésta respecto de la interfase de su entorno. Se describe que el ojo humano es más sensible a estímulos luminosos de estructuras curvas que de aquellas de ángulos rectos; por lo tanto, estructuras tubulares o esféricas producirán efecto de bandas de Mach mayores que aquellas con menor curvatura. La diferencia de luminosidad debe ocurrir en una interfase bien definida para producir un contraste suficiente que genere la respuesta de banda de Mach. Si el objeto tiene mayor opacidad de lo que lo rodea y presenta un contorno convexo en relación a una envoltura cóncava, en la interfase resultará una banda de Mach negativa; una banda positiva resultará si una de las condiciones se invierte. 
Si la diferencia de contraste es excesiva o insuficiente, la banda de Mach puede estar parcial o totalmente ausente en la interfase.

Si se efectúa un análisis de luminosidad en estas bandas, se puede determinar objetivamente que no hay un resalte negativo o positivo de la luminosidad (Figura 2), pero sin embargo, son percibidas como reales por el sistema visual.

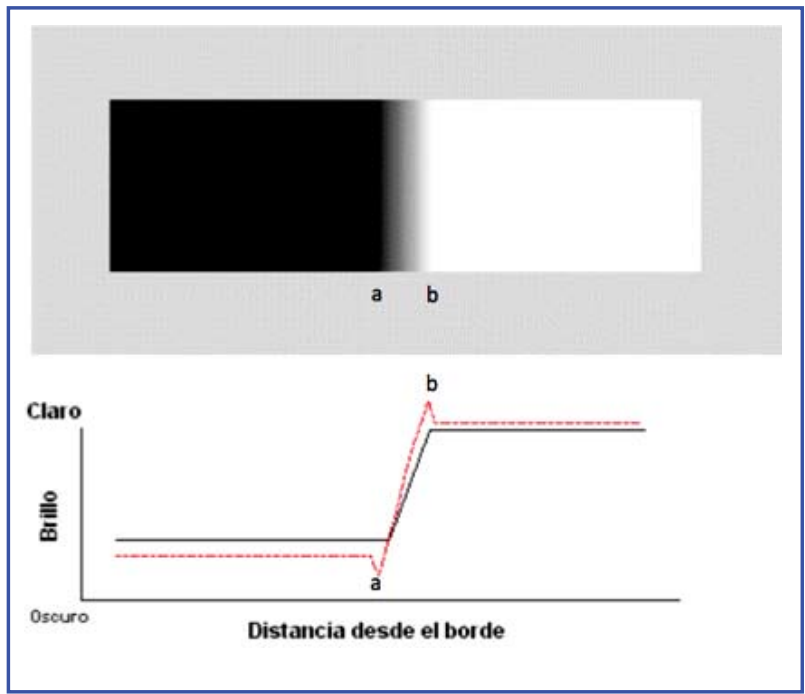

Figura 2. Determinación de densidad en una gradiente de luminosidad: La línea negra representa la medición de luminosidad por un exposímetro; no se demuestra resalte de luminosidad entre zona oscura, zona de gradiente y zona clara. La línea roja representa la percepción de luminosidad, mostrando (a) la percepción de banda oscura previa a la gradiente y (b) la banda clara al finalizar la gradiente.

\section{Teoría de las bandas de Mach}

La visualización de estas bandas estaría generada por la inhibición lateral de la retina. La existencia de inhibición lateral se ha demostrado a partir de los registros de los estudios de un cangrejo de $30 \mathrm{~cm}$ de longitud, el "Limulus" (Figura 3), que tiene múltiples elementos visuales llamados omatidios (Figura 4), de los más grandes entre los seres vivos y que pueden ser estimulados en forma individual o múltiple. En el ojo humano, a pesar de su mayor complejidad, la inhibición lateral es esencialmente similar. Un cierto número de efectos perceptivos pueden explicarse fácilmente a partir de la inhibición lateral; uno de ellos corresponde a las "bandas de Mach".

La inhibición lateral de la retina es un feedback negativo para prevenir la estimulación excesiva de un elemento único en la retina, que se basa en que la estimulación en una unidad produce una respuesta neural determinada que disminuye si otras unidades vecinas son estimuladas simultáneamente. La respuesta dependerá del número de elementos estimulados, la distancia entre los elementos y el grado de cambio de la intensidad de la luz entre dos elementos. Mientras más cerca se encuentren dos receptores habrá mayor inhibición e inversamente, mientras más alejados estén menor será la inhibición. La estimulación uniforme de los elementos visuales produce inhibición homogénea ${ }^{(1)}$ y por el contrario, los receptores adyacentes a la zona de cambio brusco de estímulo luminoso producen una sobrerespuesta (Figura 5). Este proceso de inhibición lateral nos permite mejorar el contraste de los objetos, dándole realce a los bordes $^{(2)}$.

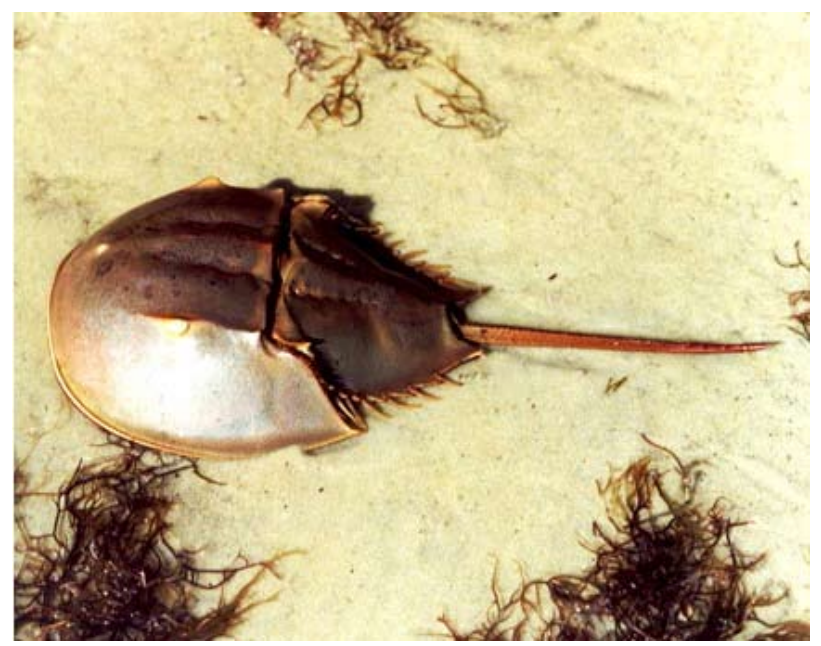

Figura 3. Cangrejo Limulus, especie en las que se han realizado experiencias de inhibición lateral de la retina.

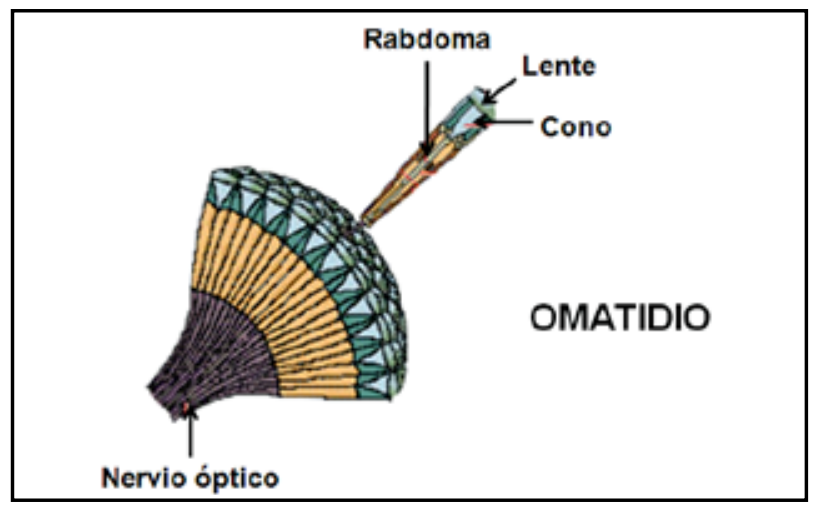

Figura 4. Esquema del ojo del Limulus.

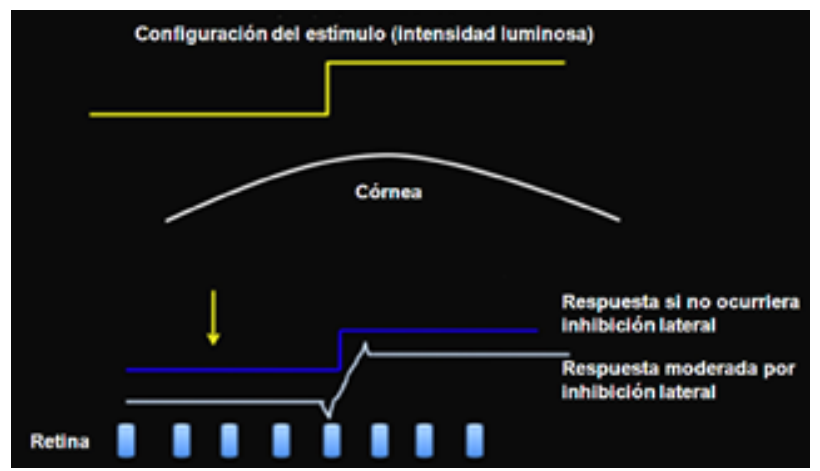

Figura 5. Esquema de la retina del Limulus y su excitación en escalón (adaptado de (1)). 
En la retina humana existen 5 tipos principales de células: Receptores(R), Bipolares(B), Ganglionares $(G)$, Horizontales $(H)$ y Amacrinas $(A)$, que se caracterizan por:

- Los tipos $\mathrm{H}$ y $\mathrm{A}$ no transmiten señales al cerebro; transmiten señales lateralmente a través de la retina.

- Las células horizontales conectan receptores con receptores.

- Las células amacrinas conectan ganglionares con bipolares.

- Las células horizontales y amacrinas pueden potencialmente transmitir esta inhibición a través de nuestra retina(2).

\section{Aplicaciones prácticas de las Bandas de Mach Banda de Mach positiva}

Este efecto se produce cuando existe una interfase entre una estructura cóncava de alta densidad y una convexa de baja densidad. En el tórax, la línea paraespinal posterior es un ejemplo de lo anterior donde la interfase entre el pulmón convexo de baja densidad y la grasa paraespinal cóncava de mayor densidad produce una banda de Mach positiva (Figura 6) ${ }^{(3)}$; otro ejemplo a nivel torácico corresponde a la banda positiva que se produce en la interfase entre un derrame pleural y el pulmón aireado adyacente (Figura 7).

Cuando visualizamos un neumotórax con pulmón adyacente de transparencia normal, se puede observar una línea opaca que corresponde a la pleura visceral y no a una banda de Mach positiva (Figura 8).
Figura 7. Mach (+) por el borde del derrame pleural.

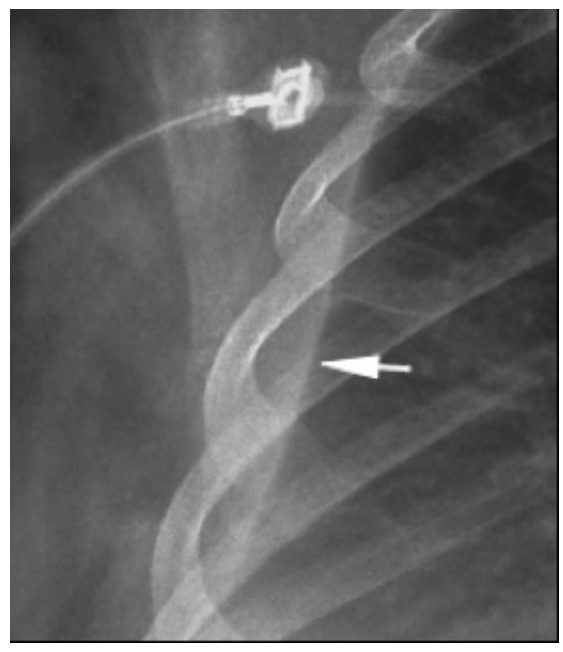

Figura 8. Falso Mach (+) de línea pleural en neumotórax.

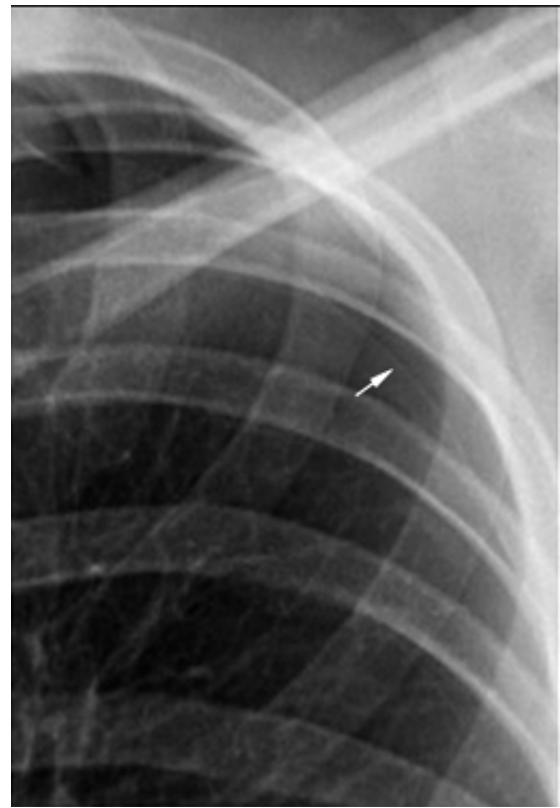

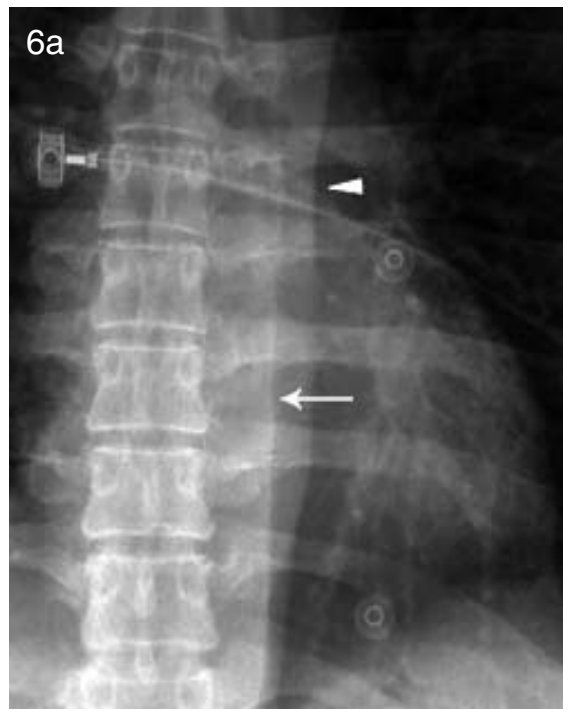

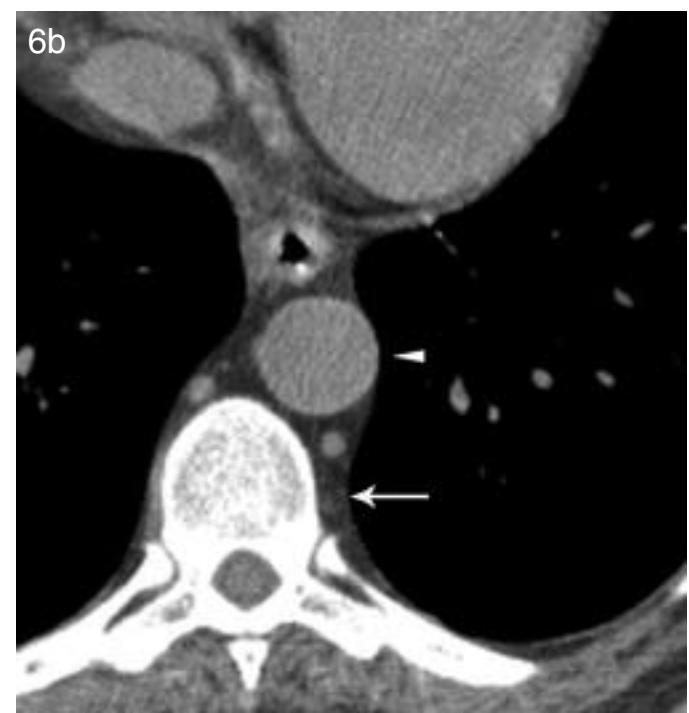

Figura 6. a) Demostración del efecto Mach (+) en línea paraespinal y Mach (-) en contorno de la aorta. b) En TC se observan los sitios en que se origina el fenómeno en la Rx: cabeza de flecha en interfase de pulmón y aorta; flecha blanca en interfase entre pulmón y grasa paraespinal. 


\section{Banda de Mach negativa}

Se originan al contactar una estructura convexa de alta densidad con una cóncava de baja densidad, en una imagen radiológica. Podemos encontrar esta situación entre el borde convexo de la aorta de alta densidad y el pulmón cóncavo, de baja densidad (Figura 6) simulando un neumomediastino, por lo que es importante conocer este efecto; la ausencia de una línea opaca, que típicamente se observa en el neumomediastino, puede permitir la diferenciación con una banda de Mach negativa ${ }^{(4)}$. Una situación similar ocurre en la interfase entre el contorno cardíaco izquierdo y el pulmón cóncavo adyacente (Figura 9).

La interfase entre un pliegue cutáneo y el aire produce una banda de Mach negativa, que no debe confundirse con un neumotórax (Figura 10); los pliegues se diferencian porque generalmente aparecen en pares o tríos, cruzan la línea media o sobrepasan el borde costal lateral|(5).

En ocasiones, puede existir la combinación de un neumotórax con un foco de condensación adyacente, lo que también producirá una banda de Mach negativa (Figura 11).

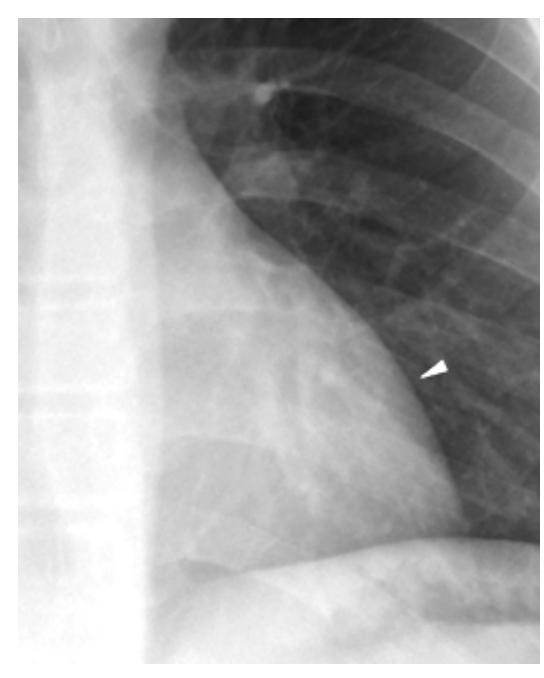

Figura 9. Banda de Mach (-) por el borde externo de la silueta cardíaca. También se forma una banda negativa por el borde superior del hemidiafragma.

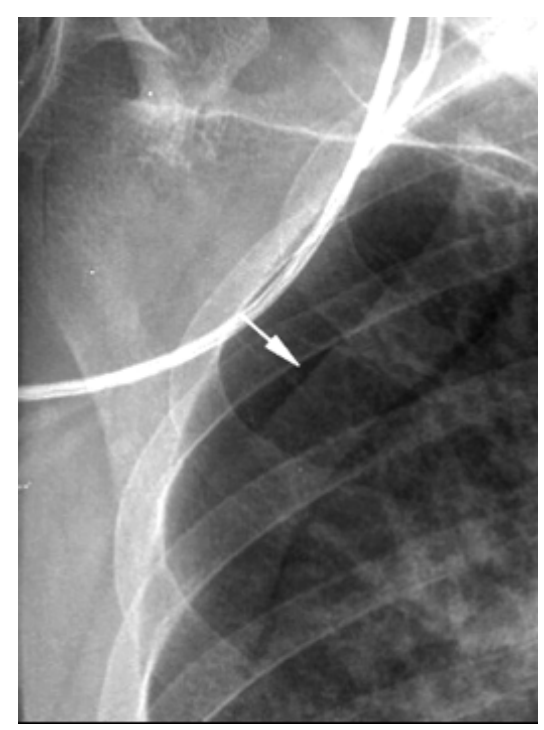

Figura 10. Mach (-) por el borde externo del pliegue cutáneo. Este efecto descarta la sospecha de neumotórax (1).
Figura 11. Mach (-) por el borde externo de la condensación, contrapuesto con un neumotórax.

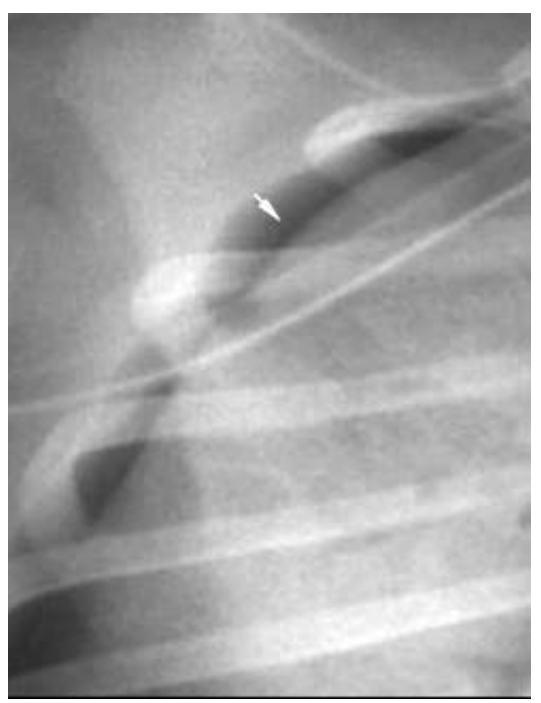

\section{Conclusiones}

Las Bandas de Mach se perciben ópticamente por un proceso psicofisiológico que se origina en la interfase entre objetos de distintas densidades, mediante un proceso de inhibición lateral en la retina que tiene como fin mejorar el contraste en los objetos dando mayor realce en los bordes; estas bandas no son físicamente reales.

Estas bandas pueden estar presentes en los exámenes radiológicos simples, como por ejemplo radiografía de tórax, y también en los topogramas de TC.

El adecuado reconocimiento de las bandas de Mach, tanto positivas como negativas, nos ayudará a la interpretación correcta del examen y, en algunas situaciones, servirá de herramienta para el diagnóstico diferencial.

\section{Bibliografía}

1. Chasen MH. Practical Applications of Mach Band Theory in Thoracic Analysis. Radiology 2001; 219(3): 596-610.
2. Guyton \& Hall. Tratado de Fisiología Médica. (11ed). Capítulo 10, Pag. 635-636. Editorial Elsevier Saunders, 2006.

3. Gibbs J, Chandrasekhar Ch, Ferguson E, Oldham S. Lines and Stripes: Where Did They Go? 10.1148/ rg.271065073 RadioGraphics 2007; 27: 33-48.

4. Zylak CM, Standen J, Barnes G, Zylak CJ. Pneumomediastinum Revisited. Radiographics 2000; 20: 1043-1057.

5. Tocino I. Pneumothorax in the supine patient: Radiographic anatomy. Radiographics 1985; 5: 557-586.

6. E. Bruce Goldstein. Sensación y percepción, 6 a edición. Capítulo 2, pag. 66. Editorial Thompson 2005. 